\title{
Comparative Analysis of Technical Parameters of Different Knitted Structures (Jersey, Pique and Lacoste) with or without Lycra/Spandex
}

\author{
Faridul Islam Ovi1,2,3, Rownak Jahan Shova ${ }^{4}$ \\ ${ }^{1}$ Department of Wet Process Engineering, Shahid Abdur Rab Serniabat Textile Engineering College, Barishal, Bangladesh \\ ${ }^{2}$ Textile Industry Management under SEIP, Bangladesh University of Textiles, Tejgaon, Dhaka, Bangladesh \\ ${ }^{3}$ Amazing Fashion Limited, National University, Gazipur, Bangladesh \\ ${ }^{4}$ Department of Apparel Manufacturing Engineering, Shahid Abdur Rab Serniabat Textile Engineering College, Barishal, \\ Bangladesh \\ Email: ovislam1612@gmail.com,rownakjahan1612@gmail.com
}

How to cite this paper: Ovi, F.I. and Shova, R.J. (2022) Comparative Analysis of Technical Parameters of Different Knitted Structures (Jersey, Pique and Lacoste) with or without Lycra/Spandex. Journal of Textile Science and Technology, 8, 68-87. https://doi.org/10.4236/jtst.2022.81007

Received: January 10, 2022

Accepted: February 25, 2022

Published: February 28, 2022

Copyright $\odot 2022$ by author(s) and Scientific Research Publishing Inc. This work is licensed under the Creative Commons Attribution International License (CC BY 4.0).

http://creativecommons.org/licenses/by/4.0/

\begin{abstract}
The relationship of Count, GSM (Gram per Square meter), and Stitch Length, Shrinkage\%, spirality\% and pilling are discussed by this paper among Pique, Lacoste and Jersey knit structures with or without Lycra " $3 \%-5 \%$ ". For this research, we used different knitted structures, yarn count and stitch length and for finding data different types of $\mathrm{m} / \mathrm{c}$ are used which are discussed later. This study is based on finish GSM of Pique, Lacoste and Jersey Knit Fabrics where various kinds of yarn count, GSM and Stitch length are used to justify the experiment. The relation of count and Stitch length with GSM is inversely proportional. When count and Stitch length increases, GSM decreases which is proved through this paper by using both the expected and experimental data. For writing this paper we have distinguished both the theoretical as well as practical implications from the industry and tried to find out adjustable points of fabrics Count, GSM and Stitch Length along with Shrinkage\%, Fabric width, Spirality. This paper provides a reliable result which will help selection of count, GSM and Stitch length for making of Pique, Lacoste and Jersey knit fabrics with or without Lycra maintaining the Shrinkage\%, spirality\%, pilling grade in an acceptable limit. This paper logically shows that GSM, shrinkage and spirality vary when structure, yarn count, and stitch length vary.
\end{abstract}

\section{Keywords}

Lycra, Jersey \& Pique \& Lacoste, Count \& GSM with Stitch Length, 
Shrinkage\% \& Pilling Grade with Spirality\%, Fabric Finish Width

\section{Introduction}

Knitting is a method of creating fabrics by transforming continuous strands of yarn into a series of interlocking loops, which are formed from a single yarn or from many yarns, each row of such loops hanging from the one immediately preceding it with the help of needles [1]. Knitted fabrics are classified into two main groups; weft and warp knitted fabrics [1]. The formation method of weft knitted can be flat and tubular [2]. In a weft knitted structure, horizontal raw of loops can be made by using one thread and the thread runs in a horizontal direction. Weft knitted loops tend to distort easily under tension and yarn can freely flow from one loop to another that is under greater elastic recovery properties. Change of yarn by horizontal striping is another major weft knitting patterning technique [3]. In a warp knitted structure, each loop in the horizontal direction is made from a different thread and the no of threads used to produce such fabrics is at least equal to the number of loops in horizontal raw. In warp knitting, the threads run thoroughly in a vertical direction. Warp knitted fabrics are not easy to unravel but these fabrics are not as elastic as weft knitted fabrics. The production of knitted fabrics is dependent on the specification of the fabrics [4]. The production of knit fabrics with spandex can be made by using a full feeder or half feeder Spandex. When the cotton and spandex yarn are knitted side by side in every course, the method is called the full feeder. When the spandex is used in alternating courses, the method is known as a half feeder. The knit items which are produced by using full feeder spandex are more compact than the items which are produced by using half feeder spandex. For the specification of knit fabrics count, GSM and stitch Length are mainly considered [5]. Weft knitted elastic fabrics are produced by the combination of cotton-spandex yarn in a circular knitting machine which have a great response and gain their original size and shape due to physical extension by any part of human body [6]. Knitted structures have a significant impact on the GSM, Shrinkage and spirality. Same count yarn is not used to produce the same GSM for different structures. When structures change, yarn count changes for producing specific GSM fabrics. Again Single jersey structure has more shrinkage tendency than Lacoste or Pique structure which are realized by this paper. Lower GSM fabric or finer count yarn has more shrinkage or spirality tendency than higher GSM fabric. Stitch length has a great impact on the GSM, Shrinkage and Spirality. GSM decreases with an increase in stitch length. More tight stitch length (than required) creates a hole in the fabric and is also responsible for increasing shrinkage and spirality.

\section{Jersey Fabrics}

There are two main forms of jersey fabrics, Plain knit and double knit jerseys. The simplest and most basic structure is the "plain knit" which is also called 
"single knit". Plain knit is formed by the needles of only one set with all the loops intermeshed in the same direction [1]. Two pieces of single knit jerseys knitted together to produce double jersey fabrics. Jersey is a soft, smooth and stretch fabric providing comfort to the wearer. It is opaque by its knitted composition that brings the fiber closer together with no way for light to pass through. The popular items such as T-shirts, tank tops, sweatpants, sweatshirts, bed sheets, underwear, sportswear, athleisure and so on are made from jersey fabrics [7]. Chain notations (Figure 1) and cam arrangement (Figure 2) of jersey are shown below-

\section{Lacoste Fabrics}

There are two main forms of Lacoste fabrics-Single Lacoste and double Lacoste. Single Lacoste is a knit-tuck single jersey structure. One set of needle is used to produce this structure. The prominence of the design appears on the back side of the fabric. Double Lacoste is tuck-knit single jersey structure. One set of needle is used to produce this structure. The prominence of the design is near to the single Lacoste fabric [1]. The chain notation (Figure 3) and Cam arrangement (Figure 4) of single Lacoste are represented below.

The chain notation (Figure 5) and Cam arrangement (Figure 6) of double Lacoste are represented below.

\section{Pique fabrics}

Pique Knit fabric is in which interlock is knitted in the first and fourth courses, while the second and fifth courses are half-gauge jersey courses knitted on one

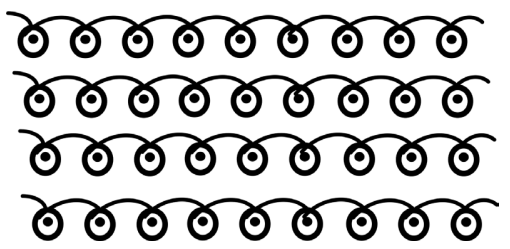

Figure 1. Chain notation Single Jersey.

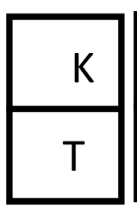

2

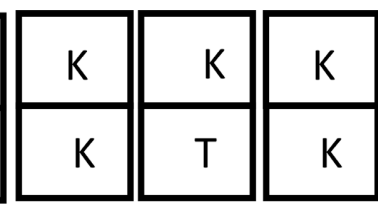

12

1

Here, $\mathrm{K}=$ Knit cam, $\mathrm{T}=$ Tuck cam.

Figure 2. Cam arragement of Single Jersey.

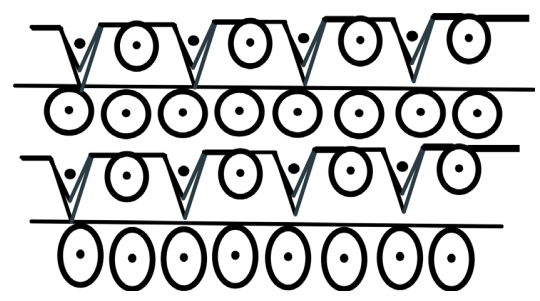

Figure 3. Chain notations of single Lacoste. 
side of the fabric, and the third and sixth courses are half gauge jersey courses knitted on the other side. Pique knit fabric is a textured fabric where the right side has a pattern like a waffle or a honeycomb shape and the wrong side is smooth. There are twice as many threads on the top layer as the bottom layer. It is widely used such as polo shirts, dresses, tennis skirts, golf wear and so on [8] [9]. The design chain notation (Figure 7) and Cam arrangement (Figure 8) are shown below.

\section{Spandex or Lycra}

Spandex is a synthetic elastic fabric typically known as lycra. It is polyurethane-

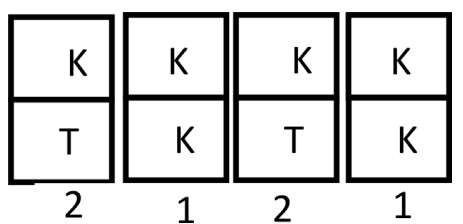

Figure 4. Cam arrangement of single Lacoste.

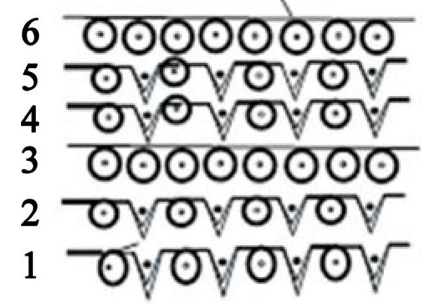

Figure 5. Chain notation of double Lacoste.

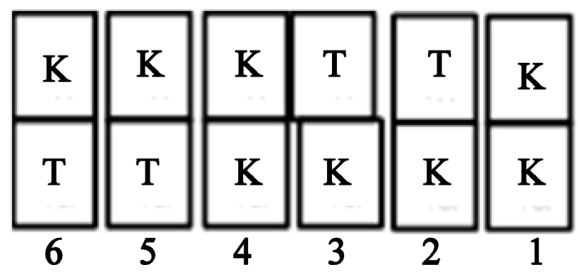

Figure 6. Cam arrangement of double Lacoste.

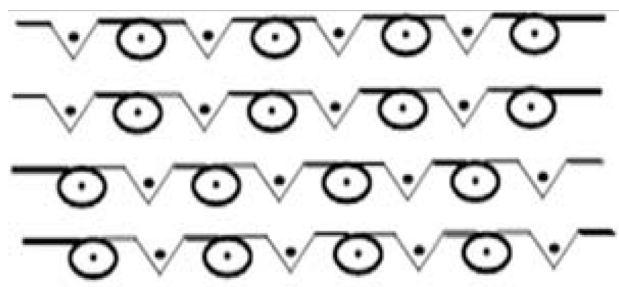

Figure 7. Chain notation of Pique.

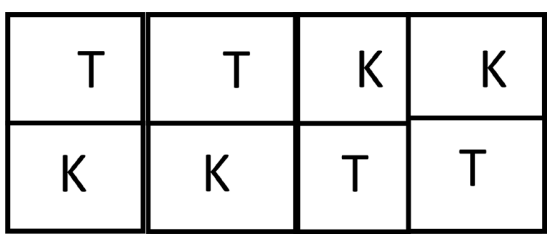

Figure 8. Cam arrangement of Pique. 
Polyurea. Spandex is never used alone and is always blended with other fibers [10]. Garments made of $100 \%$ lycra are not very popular instead a certain percentage (say "3\% - 10\%") [11]. Small amount of spandex is only required to add the properties of it into the fabrics. Trousers use around $2 \%$ to add to the comfort and fit, with the highest percentages being used in swimwear, corsetry, or sportswear reaching "15\% - 40\%" Lycra [10]. The specialty of the spandex fiber is the very high extensibility (upto-400\%) [11].

\section{The reason for using Spandex}

The elastic recovery and extension of fabrics is an important parameter for functional wear, such as, sportswear for providing freedom of body movement by reducing the fabrics resistance to body stretch [12] [13]. A body movement may extend the body skin by about $50 \%$ and the fabrics must adjust the stretch and recover on relaxation. The elasticity of a garment is provided by stretch fiver, yarn and fabrics [12] [13]. Bilateral elastic fabrics spandex provides great comfort and free movement on both sides. It can improve the fabric's hand feel, drape, and crease recovery ability. The fabrics are more elegant and less prone to distortion.

\section{Methods and Materials}

\subsection{Materials}

Fabrics were knitted with $100 \%$ cotton and with $95 \%$ cotton with 5\% Lycra. The fabrics were dyed and finished at Amantex Limited, Boiragirchala, Sreepur, Gazipur.

Sample structures: Plain Single Jersey, Lycra Single Jersey, Single Pique, Lycra Single Pique, Double Pique, Lycra Double Pique, Single Lacoste, Lycra Single Lacoste, Double Pique, Lycra Double Pique. The representation of produced Lacoste (Picture 1); Pique (Picture 2) and Single jersey (Picture 3) are shown

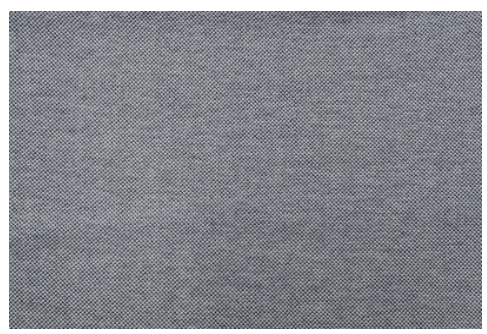

Picture 1. Lacoste.

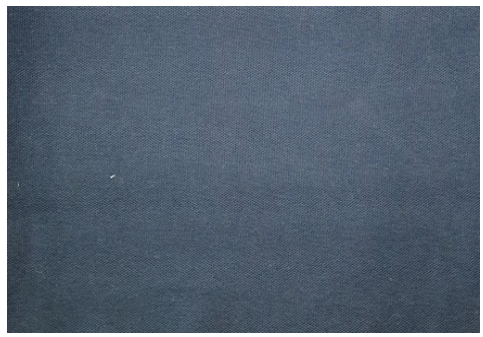

Picture 2. Pique. 


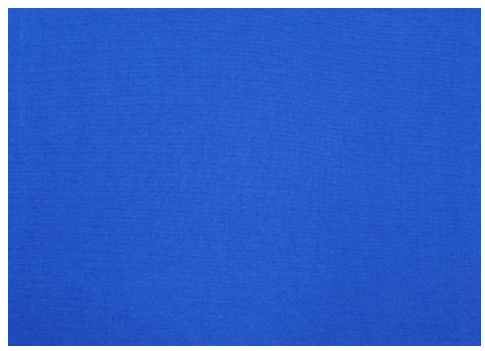

Picture 3. Single jersey.

Table 1. Knitting machine specification.

\begin{tabular}{ll}
\hline Parameters & Details \\
\hline Type & Single Jersey Circular Machine \\
Manufacturer name & Precision Fukuhara Works Ltd. \\
Country & Japan \\
Model No. & VXC-3S \\
Machine Dia & $30-36 ”$ \\
Gauge & $24 \mathrm{G}$ \\
Feeder No. & 90 \\
Machine Speed-RPM & 22 \\
\hline
\end{tabular}

in the pictue.

Knitting machine: Circular weft knitting machine is used for producing different types of fabrics. The machine parameters are shown in Table 1.

Yarn grade tester:

Type

Yarn Grade Tester

Manufacturer name

SDL International

Country

England

Test Name

Yarn grade assessment

Test Method

International method

Yarn: All the structures were produced from three types of yarn $\left(26^{\mathrm{s}}, 30^{\mathrm{s}}\right.$ and $34^{\mathrm{s}}$ ) with parameters shown in Tables 2-4. Manufacturer of all the yarn are:

Electric balance:

Type

KERN Electronic Balance

Manufacturer name

Kern \& Sohn GmbH

Country

Germany

Model No.

EG 300-3M

Washing M/C:

Type

Electrolux Washcator

Manufacturer name

Country

SDL International

Model No.

England

Test Name

FOM 71 CLS

Method Test

Dimensional stability, spirality, appearance after wash ISO 6330, BS EN ISO 26330 
Pilling Tester:

Type Orbitor Pilling \& Snaging Tester

Manufacturer name James H. Heal \& Co. Ltd

Table 2. Nominal count 26S combed. Manufacturer-DCM nouvelle Ltd. and Lot no-12.

\begin{tabular}{ll}
\hline Parameters & Details \\
\hline Actual Count & 25.93 \\
CV\% & 0.97 \\
Lea Strength & 107.37 \\
Strength CV\% & 3.86 \\
CLSP & 2780 \\
Elongation & 3.16 \\
Elongation CV\% & 8.86 \\
TPI (Single/Doule) & 13.82 \\
TM & 3.5 \\
Thin (-50\%)T/Km & 0.3 \\
Thick (+50\%)/Km & 13.8 \\
Neps (+200\%)/Km & 27.3 \\
Hairiness & 7.6 \\
Moisture Content \% & 6.99 \\
\hline
\end{tabular}

Table 3. Nominal count 30S combed. Manufacturer-S Raja exports and Lot no-30226.

\section{Parameters}

Actual Count

$\mathrm{CV} \%$

Lea Strength

Strength CV\%

CLSP

Elongation

Elongation CV\%

TPI (Single/Doule)

TM

Thin (-50\%)T/Km

Thick $(+50 \%) / \mathrm{Km}$

Neps $(+200 \%) / \mathrm{Km}$

Hairiness

Moisture Content \%

\section{Details}

30.06

1.19

102.35

4.05

3076

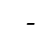

18.92

3.45

0

16.5

47

5.18

6.99 
Table 4. Nominal count $34 \mathrm{~S}$ combed. Manufacturer-Sagar manufacturer and Lot no$2131 \mathrm{C} 34020$

\begin{tabular}{ll}
\hline Parameters & Details \\
\hline Actual Count & 34.06 \\
CV\% & 1.15 \\
Lea Strength & - \\
Strength CV\% & - \\
CLSP & - \\
Elongation & 4.61 \\
Elongation CV\% & 8.31 \\
TPI (Single/Doule) & 21.48 \\
TM & 3.68 \\
Thin $(-50 \%)$ T/Km & 0 \\
Thick (+50\%)/Km & 11.8 \\
Neps (+200\%)/Km & 42.5 \\
Hairiness & 4.51 \\
Moisture Content \% & 6.99 \\
\hline
\end{tabular}

$\begin{array}{ll}\text { Country } & \text { England } \\ \text { Test Name } & \text { Pilling Resistance Test } \\ \text { Test Method } & \text { BS EN ISO 12945-1, BS } 5811 \\ \text { Fabric Appearance Tester: } \\ \text { Type } & \text { Marks \& Spencer Holoscope } \\ \text { Test Name } & \text { Measure the fabric appearance }\end{array}$

\subsection{Methods}

GSM calculation of Fabric: GSM (Gram Per Square Meter) cutter is used for cutting sample fabric (round, area $100 \mathrm{~cm}^{2}=1 \mathrm{~m}^{2}$ ) then weight is taken by electric balance. In this way, we get the sample fabric weight in gram per square meter according to ISO3801.

Stitch length: Yarn length in millimeter for one loop is known as stitch length. For measuring, first counted 100 (hundred) wales and identified both ends and then the yarn was unraveled, straightened and measured in mm. Stitch length was measured by following formula.

$$
\text { Stitch length }=\frac{\text { Length of } 100 \text { wales in } \mathrm{mm}}{\text { Total no. of wales }(100)}
$$

Thickness measurement of fabric: This test was carried out according to ISO 5084, ISO 9073 by using Digital Thickness tester.

Measuring (Shrinkage \& Spirality):

Sample: 
- Two piece of $50 \mathrm{~cm} \times 50 \mathrm{~cm}$ (Figure 9) fabric is taken for test. Procedure:

a) Conditioning: Put the sample in table for $4 \mathrm{hrs}$ for conditioning before starting test.

b) Cut the sample $50 \times 50 \mathrm{~cm}$ \& benchmark should be $35 \times 35 \mathrm{~cm}$ (Figure 9). Stitch the sample ( 3 sides) by over lock sewing machine.

c) Put sample in washing machine and run according to buyer's choice.

d) Drying: All Buyers' requirement is tumble Dry except ECHO SCOURING is flat dry.

\section{Shrinkage Test Calculation}

$$
\text { Shrinkage } \%=\frac{(\text { Before wash length }- \text { After wash length }) \times 100}{\text { Before wash length }}
$$

\section{Spirality test calculation:}

$$
\begin{gathered}
S=\left(S_{1}+S_{2}\right) / 2 \\
\text { Spirality }=(S+S \times L) / 100 .
\end{gathered}
$$

Suppose,

$S_{1}=$ The right side distance of the specimen from the stitch line after wash.

$\mathrm{S}_{2}=$ The left side distance of the specimen from the stitch line after wash.

$\mathrm{L}=$ Length before wash.

Pilling test of fabric: Little balls of fiber formed on the surface of fabric that is caused by abrasion in wear are known as pilling. For this test, sample size 5 inch $\times$ 5 inch (four pcs) was used and seam allowance of $12 \mathrm{~mm}$ was marked on the back of each square where two samples parallel to the warp direction and remaining two parallel to the weft direction. Samples were folded face to face and a seam was sewn on the marked line. Each sample turned inside out and $6 \mathrm{~mm}$ cut off (Figure 10). Each end of it thus removes any sewing distortion. Fabric tubes were mounted on rubber tubes. Each loose end taped with poly vinyl chloride tape. All of samples were placed in one pilling box and tumbled together in a cork

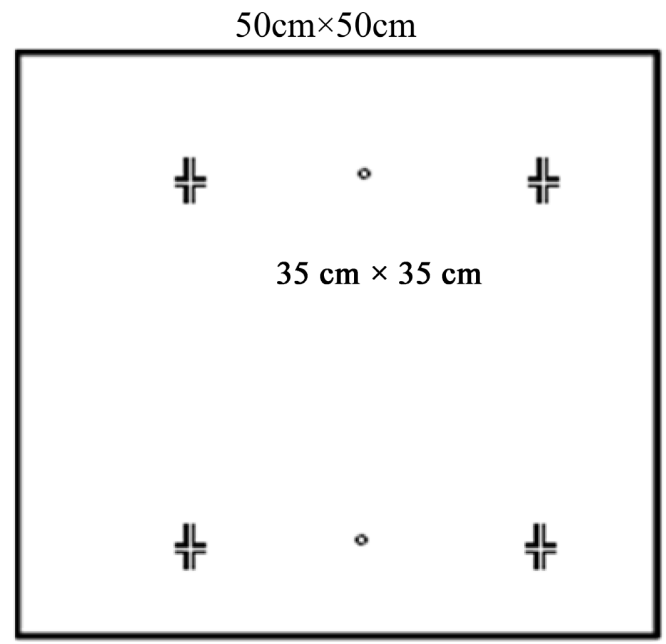

Figure 9. Test template. 


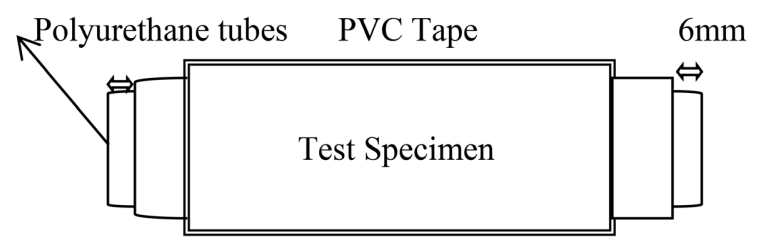

Figure 10. Preparation of a pilling sample.

Table 5. Pilling rating with description.

\begin{tabular}{cc}
\hline Rating & Description \\
\hline 5 & No change \\
4 & Slight change \\
3 & Moderate change \\
2 & Significant change \\
1 & Severe change \\
\hline
\end{tabular}

lined box. After revolutions samples were removed from the tubes and viewed using oblique lighting.

The samples are given a rating between 1 and 5 (Table 5) which indicates pilling quality of specific structure.

Calculation finish width of Lycra knitted fabric:

In case of lycra fabric, we need to find out how much width we need to keep after the heat set. If we know how much Grey GSM, Grey Width and Finish GSM will be required for our fabric, then we can easily find out the width of lycra fabric keeping finish GSM right.

Finish width $=($ Grey GSM $\times$ Grey Width $\times 1.1 \times 0.9 \times 0.86) \div$ Finish GSM

\section{Results \& Discussions:}

The variation in Structures, raw material, yarn count, stitch length, depth of color and elastomeric yarn ratio influence the structural properties and performance of the knitted fabrics. All the experiment was performed at $20^{\circ} \mathrm{C} \pm 2{ }^{\circ} \mathrm{C}$ and $65 \% \pm 2 \%$. No tension was applied during measuring GSM, Shrinkage $\%$, Spirality\%.

In this experiment, all the operations were carried out for average color (color depth " $2 \%-4 \%$ ").

\section{Knitted fabrics structure with $100 \%$ Cotton:}

Table 6 and Table 7 below show expected data and experimental data for different knitted structures when yarn count $26^{\mathrm{s}} \mathrm{kept}$ constant.

For fixed count 26 s, fixed weight fabrics can be produced without affecting physical properties much that the table shows. From data in Table 6, it is clear that fabric structure has a huge impact on the fabric weight and thickness. For the same count, different knitted fabrics vary with GSM and Stitch Length.

No major variation was found comparing Table $6 \&$ Table 7. All the parameters were in tolerance level but in case double pique, the shrinkage\% was slightly 
high where spirality and pilling grade was good. Observing Table 7, it was found that single structures were more prone to show higher shrinkage and spirality than double structures.

Table 8 and Table 9 below show expected data and experimental data for different knitted structures when yarn count $30^{\mathrm{s}}$ kept constant.

The statistical data (Table 8) shows that GSM, Stitch length, thickness are varying with changing knitted structures for fixed count $30^{\mathrm{s}} .30^{\mathrm{s}}$ is suitable for

Table 6. Expected data.

\begin{tabular}{|c|c|c|c|c|c|c|c|c|}
\hline Structures & Count & Target GSM & S.L cm & Thickness mm & Shrinkage\% & Spirality\% & Pilling grade & Dia in inch \\
\hline Single Jersey-SJ & $26^{\mathrm{s}}$ & $160-170$ & 2.80 & 0.187 & $\begin{array}{c}\mathrm{L}=-3 \%-4 \% \\
\mathrm{~W}=-3 \%-4 \%\end{array}$ & $1-2 \%$ & $4-5$ & 62 \\
\hline Single Pique-SP & $26^{\mathrm{s}}$ & $180-190$ & 2.66 & 0.232 & $\begin{array}{c}L=-4 \%-5 \% \\
W=-3 \%-4 \%\end{array}$ & $1 \%-2 \%$ & $4-5$ & 75 \\
\hline Double Pique-DP & $26^{\mathrm{s}}$ & $200-210$ & 2.70 & 0.277 & $\begin{array}{c}\mathrm{L}=-4 \%-5 \% \\
\mathrm{~W}=-3 \%-4 \%\end{array}$ & $1 \%-2 \%$ & $4-5$ & 80 \\
\hline Single Lacoste-SL & $26^{\mathrm{s}}$ & $190-200$ & 2.65 & 0.235 & $\begin{array}{c}\mathrm{L}=-4 \%-5 \% \\
\mathrm{~W}=-3 \%-4 \%\end{array}$ & $1 \%-2 \%$ & $4-5$ & 75 \\
\hline Double Lacoste-DL & $26^{\mathrm{s}}$ & $220-230$ & 2.55 & 0.302 & $\begin{array}{c}\mathrm{L}=-4 \%-5 \% \\
\mathrm{~W}=-3 \%-4 \%\end{array}$ & $1 \%-2 \%$ & $4-5$ & 80 \\
\hline
\end{tabular}

Table 7. Experimental data.

\begin{tabular}{ccccccccc}
\hline Structures & Count & Found GSM & S.L cm & Thickness mm & Shrinkage $\%$ & Spirality\% & Pilling grade & Dia in inch \\
\hline SJ-1 & $26^{\mathrm{s}}$ & 159 & 2.80 & 0.189 & $\mathrm{~L}=-4 \%, \mathrm{~W}=-5 \%$ & $2 \%$ & 2.5 & 62 \\
SP-1 & $26^{\mathrm{s}}$ & 195 & 2.66 & 0.237 & $\mathrm{~L}=-5 \%, \mathrm{~W}=-3 \%$ & $2 \%$ & 3 & 74 \\
DP-1 & $26^{\mathrm{s}}$ & 207 & 2.70 & 0.278 & $\mathrm{~L}=-5 \%, \mathrm{~W}=-4.5 \%$ & $2.2 \%$ & 3 & 81 \\
SL-1 & $26^{\mathrm{s}}$ & 193 & 2.65 & 0.233 & $\mathrm{~L}=-6.5 \%, \mathrm{~W}=-4 \%$ & $1 \%$ & 3.5 & 75 \\
DL-1 & $26^{\mathrm{s}}$ & 240 & 2.55 & 0.312 & $\mathrm{~L}=-6 \%, \mathrm{~W}=-2 \%$ & $2 \%$ & & 79 \\
\hline
\end{tabular}

Table 8. Expected data.

\begin{tabular}{|c|c|c|c|c|c|c|c|c|}
\hline Structures & Count & Target GSM & S.L cm & Thickness mm & Shrinkage\% & Spirality\% & Pilling grade & Dia in inch \\
\hline SJ & $30^{\mathrm{s}}$ & $140-150$ & 2.67 & 0.167 & $\begin{array}{c}\mathrm{L}=-3 \%-4 \% \\
W=-5 \%-6 \%\end{array}$ & $1 \%-2 \%$ & $4-5$ & 60 \\
\hline SP & $30^{\mathrm{s}}$ & $150-160$ & 2.55 & 0.195 & $\begin{array}{c}L=-5 \%-6 \% \\
W=-3 \%-4 \%\end{array}$ & $1 \%-2 \%$ & $4-5$ & 73 \\
\hline $\mathrm{DP}$ & $30^{\mathrm{s}}$ & $180-190$ & 2.60 & 0.248 & $\begin{array}{c}L=-5 \%-6 \% \\
W=-3 \%-4 \%\end{array}$ & $1 \%-2 \%$ & $4-5$ & 77 \\
\hline SL & $30^{\mathrm{s}}$ & $160-170$ & 2.45 & 0.208 & $\begin{array}{c}L=-5 \%-6 \% \\
W=-3 \%-4 \%\end{array}$ & $1 \%-2 \%$ & $4-5$ & 73 \\
\hline $\mathrm{DL}$ & $30^{\mathrm{s}}$ & $190-200$ & 2.70 & 0.276 & $\begin{array}{c}L=-5 \%-6 \% \\
W=-3 \%-4 \%\end{array}$ & $1 \%-2 \%$ & $4-5$ & 77 \\
\hline
\end{tabular}


producing specific weight fabric for specific knitted structures. Shrinkage\%, Spirality\%, Pilling grade, Hand feel are expected to be good.

Comparing statistical data between Table 6 and Table 8, it is found that GSM is decreasing with an increase in yarn count. For the increase of yarn count four $\left(26^{\mathrm{s}}-30^{\mathrm{s}}=4^{\mathrm{s}}\right.$, Higher the cotton count, finer the yarn), the amount of GSM decreasing is near twenty. As a result, heavy fabrics are produced from lower count yarn.

Table 10 and Table 11 below show expected data and experimental data for different knitted structures when yarn count $34^{\mathrm{s}}$ kept constant.

Shrinkage\% and Spirality\% are increasing with the increase of yarn count for above knitted structures.

Comparison of Experimental data among SJ-1, SJ-2 and SJ-3 from Table 7, Table 9 and Table 11 are represented in Figure 11.

Comparison of Experimental data among SP-1, SP-2 and SP-3 from Table 7, Table 9 and Table 11 are represented in Figure 12.

Table 9. Experimental data.

\begin{tabular}{ccccccccc}
\hline Structures & Count & Found GSM & S.L (cm) & Thickness $(\mathrm{mm})$ & Shrinkage\% & \multicolumn{2}{c}{ Spirality } & Pilling Dia (inch) \\
\hline SJ-2 & $30^{\mathrm{s}}$ & 146 & 2.67 & 0.189 & $\mathrm{~L}=-3 \%, \mathrm{~W}=-6 \%$ & $2 \%$ & 3 & 61 \\
SP-2 & $30^{\mathrm{s}}$ & 167 & 2.55 & 0.204 & $\mathrm{~L}=-6.7 \%, \mathrm{~W}=-4.2 \%$ & $3 \%$ & 3.5 & 75 \\
DP-2 & $30^{\mathrm{s}}$ & 184 & 2.60 & 0.244 & $\mathrm{~L}=-7 \%, \mathrm{~W}=-2 \%$ & $2 \%$ & 3.5 & 79 \\
SL-2 & $30^{\mathrm{s}}$ & 158 & 2.45 & 0.199 & $\mathrm{~L}=-7 \%, \mathrm{~W}=-5.3 \%$ & $3 \%$ & 4 & 74 \\
DL-2 & $30^{\mathrm{s}}$ & 177 & 2.70 & 0.248 & $\mathrm{~L}=-8 \%, \mathrm{~W}=-3.5 \%$ & $3 \%$ & 3.5 & 78 \\
\hline
\end{tabular}

Table 10. Expected data.

\begin{tabular}{ccccccccc}
\hline Structures & Count & Found GSM & S.L (cm) & Thickness $(\mathrm{mm})$ & Shrinkage\% & \multicolumn{2}{c}{ Spirality } & Pilling Dia (inch) \\
\hline SJ & $34^{\mathrm{s}}$ & $120-130$ & 26.2 & 0.144 & $\mathrm{~L}=-6 \%, \mathrm{~W}=-8 \%$ & $2 \%-3 \%$ & $4-5$ & 57 \\
SP & $34^{\mathrm{s}}$ & $120-130$ & 2.30 & 0.157 & $\mathrm{~L}=-7 \%-8 \%, \mathrm{~W}=-5 \%-6 \%$ & $2 \%-3 \%$ & $4-5$ & 70 \\
DP & $34^{\mathrm{s}}$ & $135-145$ & 2.35 & 0.180 & $\mathrm{~L}=-7 \%-8 \%, \mathrm{~W}=-5 \%-6 \%$ & $2 \%-3 \%$ & $4-5$ & 74 \\
SL & $34^{\mathrm{s}}$ & $120-130$ & 2.31 & 0.156 & $\mathrm{~L}=-7 \%-8 \%, \mathrm{~W}=-5 \%-6 \%$ & $2 \%-3 \%$ & $4-5$ & 70 \\
DL & $34^{\mathrm{s}}$ & $135-145$ & 2.37 & 0.179 & $\mathrm{~L}=-7 \%-8 \%, \mathrm{~W}=-5 \%-6 \%$ & $2 \%-3 \%$ & $4-5$ & 74 \\
\hline
\end{tabular}

Table 11. Experimental data.

\begin{tabular}{ccccccccc}
\hline Structures & Count & Found GSM & S.L $(\mathrm{cm})$ & Thickness $(\mathrm{mm})$ & Shrinkage $\%$ & Spirality & Pilling & Dia (inch) \\
\hline SJ-3 & $34^{\mathrm{s}}$ & 123 & 2.62 & 0.140 & $\mathrm{~L}=-6 \%, \mathrm{~W}=-9 \%$ & $2.5 \%$ & 3 & 59 \\
SP-3 & $34^{\mathrm{s}}$ & 115 & 2.30 & 0.145 & $\mathrm{~L}=-10 \%, \mathrm{~W}=-7 \%$ & $3 \%$ & 4.5 & 73 \\
DP-3 & $34^{\mathrm{s}}$ & 123 & 2.35 & 0.163 & $\mathrm{~L}=-9 \%, \mathrm{~W}=-8 \%$ & $2.4 \%$ & 4.5 & 76 \\
SL-3 & $34^{\mathrm{s}}$ & 126 & 2.31 & 0.157 & $\mathrm{~L}=-11 \%, \mathrm{~W}=-6 \%$ & $3 \%$ & 4.5 & 74 \\
DL-3 & $34^{\mathrm{s}}$ & 133 & 2.37 & 0.175 & $\mathrm{~L}=-11 \%, \mathrm{~W}=-8 \%$ & $3 \%$ & 4 & 77 \\
\hline
\end{tabular}


Comparison of Experimental data among DP-1, DP-2 and DP-3 from Table 7, Table 9 and Table 11 are shown in Figure 13.

Comparison of Experimental data among SL-1, SL-2 and SL-3 from Table 7, Table 9 and Table 11 are shown in Figure 14.

Comparison of Experimental data among DL-1, DL-2 and DL-3 from Table 7, Table 9 and Table 11 are realized from Figure 15.

The above comparison (among Figures 11-15) report shows that every individual count has as specific stitch length for producing specific weight fabric

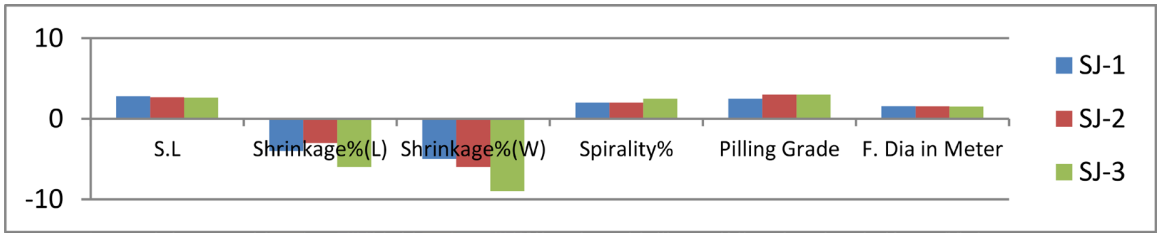

Figure 11. Yarn count \& stitch length effect on single jersey parameters.

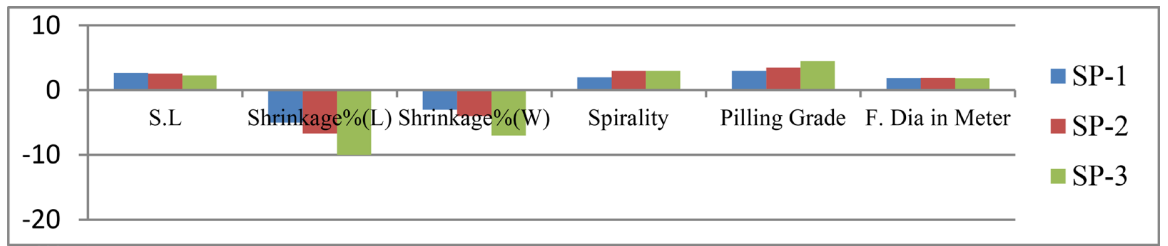

Figure 12. Yarn count \& stitch length effect on single pique structure.

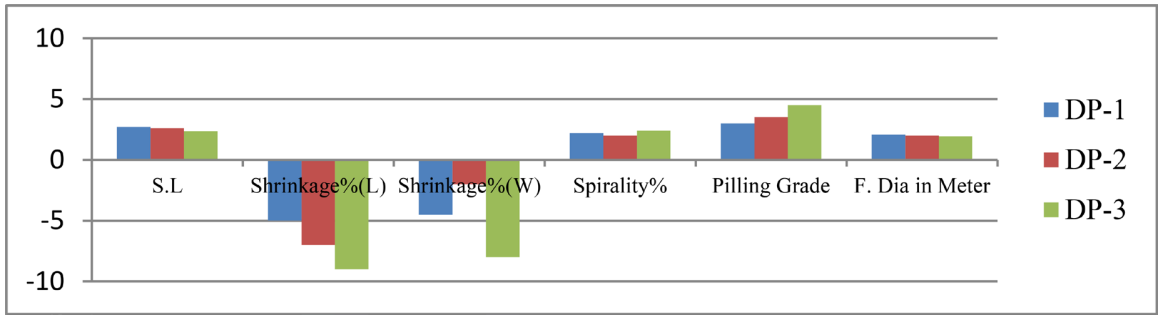

Figure 13. Yarn count \& stitch length effect on double pique structure.

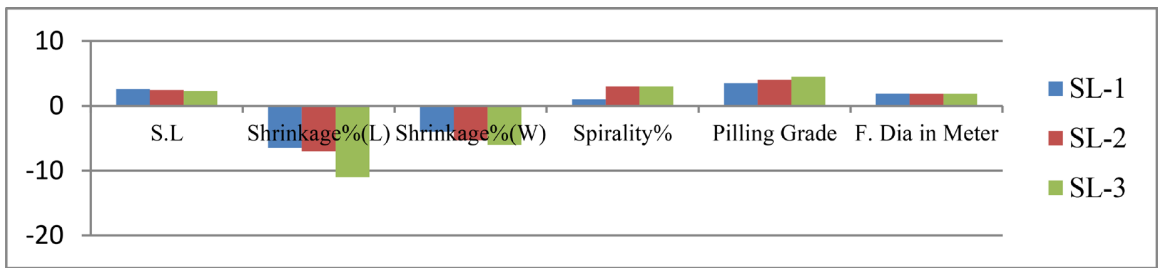

Figure 14. Yarn count \& stitch length effect on single lacoste structure.

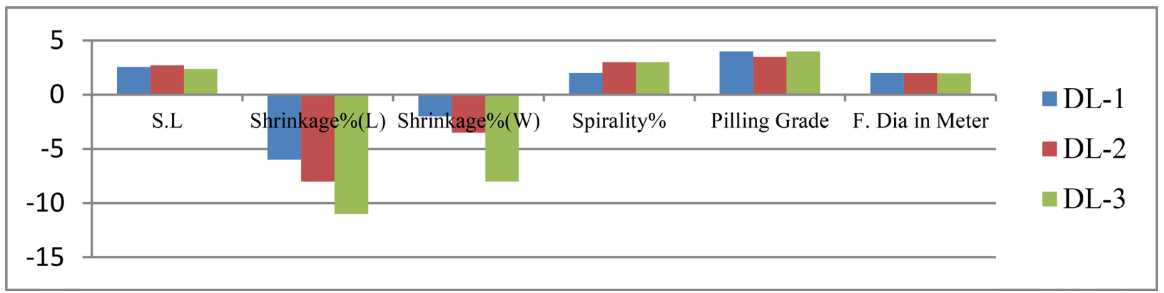

Figure 15. Yarn count \& stitch length effect on double lacoste structure. 
for maintaining standard parameter. Changing in stitch length will change the standard parameter. Pilling grade decreases and shrinkage\% increases with the increase of stitch length but spirality slightly changed. So the result, in short, is when count increases GSM decreases, Shrinkage\% increases, thickness decreases and spirality increases. Shrinkage\% and spirality\% increase with the deviation of finish dia from expected standard.

Knitted fabrics structure with 95\% Cotton and 5\% Lycra: GSM and dimensional stability are the most important parameter of Lycra related fabrics. The internal structure of the fabric is also tense due to the tension of the yarn during the making of Lycra fabric in the knitting machine. After unloading the cloth from the machine, the cloth is relived. As a result, the fabric shrinks slightly along the width. As the width decreases, the weight of the cloth increases. It is not easy to make garment if the weight of fabrics does not maintain a certain limit because there will be a shortage of fabrics due to the decrease of width. It is needed to heat set the fabrics (about $180^{\circ} \mathrm{C}$ by stenter $\mathrm{m} / \mathrm{c}$ ) to solve that problem.

The below Table 12 and Table 13 show expected data and experimental data for different knitted structures with $95 \%$ cotton \& 5\% Lycra when yarn count $26^{\circ}$ kept constant with 40d Lycra (5\%).

The knitted structures with 5\% lycra produced more heavy fabrics than without lycra for the same constant count. Half feeder lycra has been used for the lycra single jersey, lycra single pique, lycra single lacoste but lycra has been used after two feeders for the lycra double lacoste due to the knit, tuck cam arrangement. The production of lycra double pique was hardly possible due to the

Table 12. Expected Data.

\begin{tabular}{|c|c|c|c|c|c|c|c|c|}
\hline Structures & Count & $\begin{array}{c}\text { Target } \\
\text { GSM }\end{array}$ & $\begin{array}{c}\text { Stitch } \\
\text { Length in } \mathrm{cm}\end{array}$ & Finish Dia & $\begin{array}{l}\text { Thickness } \\
\text { in } \mathrm{mm}\end{array}$ & Shrinkage\% & Spirality\% & $\begin{array}{l}\text { Pilling } \\
\text { Grade }\end{array}$ \\
\hline Lycra SJ & $26^{\mathrm{s}}$ & $245-255$ & 2.96 & 65 " open & 0.306 & $\mathrm{~L}=-6 \%, \mathrm{~W}=-6 \%$ & $1-2 \%$ & $4-5$ \\
\hline Lycra SP & $26^{\mathrm{s}}$ & $250-260$ & 2.77 & 74 " open & 0.300 & $\mathrm{~L}=-7 \%, \mathrm{~W}=-7 \%$ & $1-2 \%$ & $4-5$ \\
\hline Lycra DP & $26^{\mathrm{s}}$ & \multicolumn{7}{|c|}{ Hardly produce this structure } \\
\hline Lycra SL & $26^{\mathrm{s}}$ & $245-260$ & 2.80 & 74" open & 0.305 & $\mathrm{~L}=-7 \%, \mathrm{~W}=-7 \%$ & $1-2 \%$ & $4-5$ \\
\hline Lycra DL & $26^{\mathrm{s}}$ & $260-270$ & 2.75 & 75 ” open & 0.310 & $\mathrm{~L}=-7 \%, \mathrm{~W}=-7 \%$ & $1-2 \%$ & $4-5$ \\
\hline
\end{tabular}

Table 13. Experimental data.

\begin{tabular}{|c|c|c|c|c|c|c|c|c|}
\hline Structures & Count & $\begin{array}{l}\text { Found } \\
\text { GSM }\end{array}$ & $\begin{array}{l}\text { Stitch Length } \\
\text { in } \mathrm{cm}\end{array}$ & Finish Dia & $\begin{array}{l}\text { Thickness } \\
\text { in } \mathrm{mm}\end{array}$ & Shrinkage\% & Spirality\% & $\begin{array}{l}\text { Pilling } \\
\text { Grade }\end{array}$ \\
\hline LSJ-1 & $26^{\mathrm{s}}$ & 252 & 2.96 & 65 " open & 0.308 & $\mathrm{~L}=-6.4 \%, \mathrm{~W}=-7.5 \%$ & $2.2 \%$ & 2.5 \\
\hline LSP-1 & $26^{\mathrm{s}}$ & 265 & 2.77 & 76" open & 0.305 & $\mathrm{~L}=-7.5 \%, \mathrm{~W}=-5.1 \%$ & $1 \%$ & 3.5 \\
\hline LDP-1 & $26^{\mathrm{s}}$ & \multicolumn{7}{|c|}{ Hardly produce this structure } \\
\hline LSL-1 & $26^{\mathrm{s}}$ & 256 & 2.80 & 75 " open & 0.296 & $\mathrm{~L}=-7.8 \%, \mathrm{~W}=-6.9 \%$ & $1.5 \%$ & 3.5 \\
\hline LDL-1 & $26^{\mathrm{s}}$ & 261 & 2.75 & 73" open & 0.301 & $\mathrm{~L}=-7.2 \%, \mathrm{~W}=-5 \%$ & $2 \%$ & 3.5 \\
\hline
\end{tabular}


arrangement of knit and tuck cam. Even if we produced the structure, lycra will be seen from outside and the appearance will not be desirable. The fabrics finish dia and heat set maintained for controlling shrinkage\% and spirality\% along with GSM, dimensional stability and elastic property.

Table 14 and Table 15 below show expected data and experimental data for different knitted structures with $95 \%$ cotton \& 5\% Lycra when yarn count $30^{\mathrm{s}}$ kept constant with 40d Lycra (5\%).

Table 16 and Table 17 below show expected data and experimental data for different knitted structures with $95 \%$ cotton \& 5\% Lycra when yarn count $26^{\mathrm{s}}$ kept constant with 40d Lycra (5\%).

Comparison of Experimental data among LSJ-1, LSJ-2 and L SJ-3 from Table 13, Table 15 and Table 17 are given in Figure 16.

Comparison of Experimental data among LSP-1, LSP-2 and LSP-3 from Table 13, Table 15 and Table 17 are represented in Figure 17.

Table 14. Expected data.

\begin{tabular}{|c|c|c|c|c|c|c|c|c|}
\hline Structures & Count & Target GSM & $\begin{array}{l}\text { Stitch Length } \\
\text { in } \mathrm{cm}\end{array}$ & Finish Dia & $\begin{array}{l}\text { Thickness } \\
\text { in } \mathrm{mm}\end{array}$ & Shrinkage\% & Spirality\% & $\begin{array}{l}\text { Pilling } \\
\text { Grade }\end{array}$ \\
\hline Lycra SJ & $30^{\mathrm{s}}$ & $200-210$ & 2.95 & 62 " open & 0.250 & $\mathrm{~L}=-6 \%, \mathrm{~W}=-6 \%$ & $1 \%-2 \%$ & $4-5$ \\
\hline Lycra SP & $30^{\mathrm{s}}$ & $195-205$ & 2.77 & 75 ”open & 0.235 & $\mathrm{~L}=-7 \%, \mathrm{~W}=-7 \%$ & $1 \%-2 \%$ & $4-5$ \\
\hline Lycra DP & $30^{\mathrm{s}}$ & \multicolumn{7}{|c|}{ Hardly produce this structure } \\
\hline Lycra SL & $30^{\mathrm{s}}$ & $200-210$ & 2.75 & 74 " open & 0.252 & $\mathrm{~L}=-7 \%, \mathrm{~W}=-7 \%$ & $1 \%-2 \%$ & $4-5$ \\
\hline Lycra DL & $30^{\mathrm{s}}$ & $230-240$ & 2.80 & $74 "$ open & 0.274 & $\mathrm{~L}=-7 \%, \mathrm{~W}=-7 \%$ & $1 \%-2 \%$ & $4-5$ \\
\hline
\end{tabular}

Table 15. Experimental data.

\begin{tabular}{|c|c|c|c|c|c|c|c|c|}
\hline Structures & Count & $\begin{array}{l}\text { Found } \\
\text { GSM }\end{array}$ & $\begin{array}{l}\text { Stitch Length } \\
\text { in } \mathrm{cm}\end{array}$ & Finish Dia & $\begin{array}{l}\text { Thickness } \\
\text { in } \mathrm{mm}\end{array}$ & Shrinkage\% & Spirality\% & $\begin{array}{l}\text { Pilling } \\
\text { Grade }\end{array}$ \\
\hline LSJ-2 & $30^{\mathrm{s}}$ & 206 & 2.95 & 63 " open & 0.254 & $\mathrm{~L}=-8.1 \%, \mathrm{~W}=-7.3 \%$ & $2.5 \%$ & 2.5 \\
\hline LSP-2 & $30^{\mathrm{s}}$ & 211 & 2.77 & 72 ” open & 0.341 & $\mathrm{~L}=-8.4 \%, \mathrm{~W}=-6.1 \%$ & $1.5 \%$ & 3.5 \\
\hline LDP-2 & $30^{\mathrm{s}}$ & \multicolumn{7}{|c|}{ Hardly produce this structure } \\
\hline LSL-2 & $30^{\mathrm{s}}$ & 214 & 2.75 & 73 " open & 0.260 & $\mathrm{~L}=-8.1 \%, \mathrm{~W}=-6.9 \%$ & $1.5 \%$ & 3.5 \\
\hline LDL-2 & $30^{\mathrm{s}}$ & 240 & 2.80 & 74 " open & 0.276 & $\mathrm{~L}=-8.5 \%, \mathrm{~W}=-7.2 \%$ & $2.3 \%$ & 3 \\
\hline
\end{tabular}

Table 16. Expected data.

\begin{tabular}{ccccccccc}
\hline Structures & Count & Target GSM & S.L in cm & Finish Dia & Thickness in mm & Shrinkage\% & Spirality\% & Pilling Grade \\
\hline Lycra SJ & $34^{\mathrm{s}}$ & $170-180$ & 2.85 & $58^{\prime \prime}$ open & 0.218 & $\mathrm{~L}=-6 \%, \mathrm{~W}=-6 \%$ & $1-2 \%$ & $4-5$ \\
Lycra SP & $34^{\mathrm{s}}$ & $180-190$ & 2.90 & 74 ”open & 0.210 & $\mathrm{~L}=-7 \%, \mathrm{~W}=-7 \%$ & $1-2 \%$ & $4-5$ \\
Lycra DP & $34^{\mathrm{s}}$ & & & & Hardly produce this structure & & & \\
Lycra SL & $34^{\mathrm{s}}$ & $180-190$ & 2.90 & $74^{\prime \prime}$ open & 0.225 & $\mathrm{~L}=-7 \%, \mathrm{~W}=-7 \%$ & $1-2 \%$ & $4-5$ \\
Lycra DL & $34^{\mathrm{s}}$ & $200-210$ & 2.85 & $74^{\prime \prime}$ open & 0.236 & $\mathrm{~L}=-7 \%, \mathrm{~W}=-7 \%$ & $1-2 \%$ & $4-5$ \\
\hline
\end{tabular}


Comparison of Experimental data among LSL-1, LSL-2 and LSL-3 from Table 13, Table 15 and Table 17 are shown in Figure 18.

Comparison of Experimental data among LDL-1, LDL-2 and LDL-3 from Table 13, Table 15 and Table 17 are realized from Figure 19.

The above column diagrams represent S.L, lengthwise and widthwise shrinkage\%, spirality\%, Finish width, pilling grade with respect to constant count. Each parameter is dependent on each other. Shrinkage\% and Spirality\% increase with the increase of finish width from standard. Stitch length varies with knitted structure to produce suitable fabrics. The coarser the count, the lower the shrinkage $\%$ and spirality\%. Pilling grade varies with structure but compact stitch

Table 17. Experimental data.

\begin{tabular}{|c|c|c|c|c|c|c|c|c|c|}
\hline Structures & Count & Target GSM & \multicolumn{2}{|c|}{$\begin{array}{l}\text { Stitch Length } \\
\text { in } \mathrm{cm}\end{array}$} & Finish Dia & $\begin{array}{l}\text { Thickness } \\
\text { in } \mathrm{mm}\end{array}$ & Shrinkage\% & Spirality\% & $\begin{array}{l}\text { Pilling } \\
\text { Grade }\end{array}$ \\
\hline LSJ-3 & $34^{\mathrm{s}}$ & 185 & \multicolumn{2}{|c|}{2.85} & $60 "$ open & 0.220 & $\mathrm{~L}=-7.5 \%, \mathrm{~W}=-8 \%$ & $3 \%$ & 3 \\
\hline LSP-3 & $34^{\mathrm{s}}$ & 177 & \multicolumn{2}{|c|}{2.90} & $76 "$ open & 0.209 & $\mathrm{~L}=-8.3 \%, \mathrm{~W}=-7.2 \%$ & $2.5 \%$ & 3 \\
\hline LDP-3 & $34^{\mathrm{s}}$ & \multicolumn{8}{|c|}{ Hardly produce this structure } \\
\hline LSL-3 & $34^{\mathrm{s}}$ & 184 & \multicolumn{2}{|c|}{2.90} & 77” open & 0.223 & $\mathrm{~L}=-9.1 \%, \mathrm{~W}=-4 \%$ & $2.3 \%$ & 3 \\
\hline LDL-3 & $34^{\mathrm{s}}$ & 202 & \multicolumn{2}{|c|}{2.85} & 75 " open & 0.234 & $\mathrm{~L}=-8.8 \%, \mathrm{~W}=-7.6 \%$ & $3.1 \%$ & 3.5 \\
\hline & & & \multirow{3}{*}{$\begin{array}{r}10 \\
0\end{array}$} & & & & & & \multirow{3}{*}{$\begin{array}{l}\text { LSJ-1 } \\
\text { LSJ-2 } \\
\text { LSJ-3 }\end{array}$} \\
\hline & & & & & & & -1 & - & \\
\hline & & & & S.L & Shrinkage\% & (L) Shrinkage\%(W) & Pilling grade & F. Dia in Meter & \\
\hline
\end{tabular}

Figure 16. Yarn count \& stitch length effect on Lycra single jersey structure.

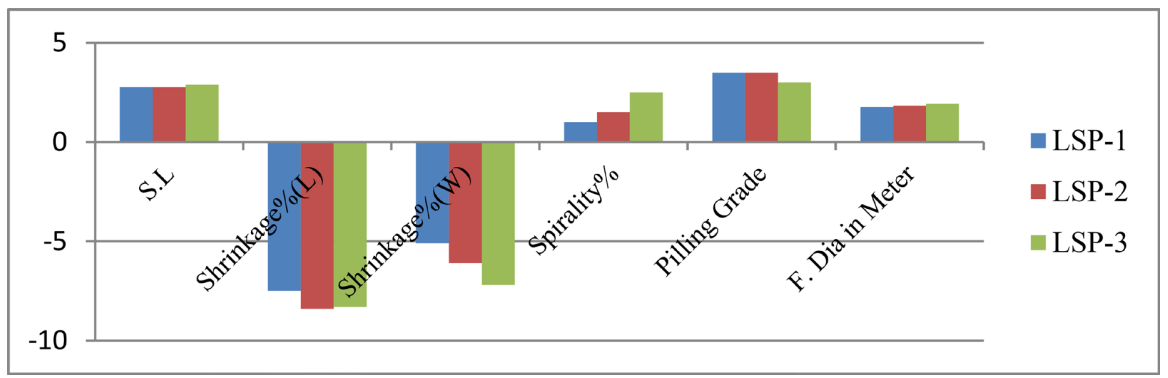

Figure 17. Yarn count \& stitch length effect on lycra single pique structure.

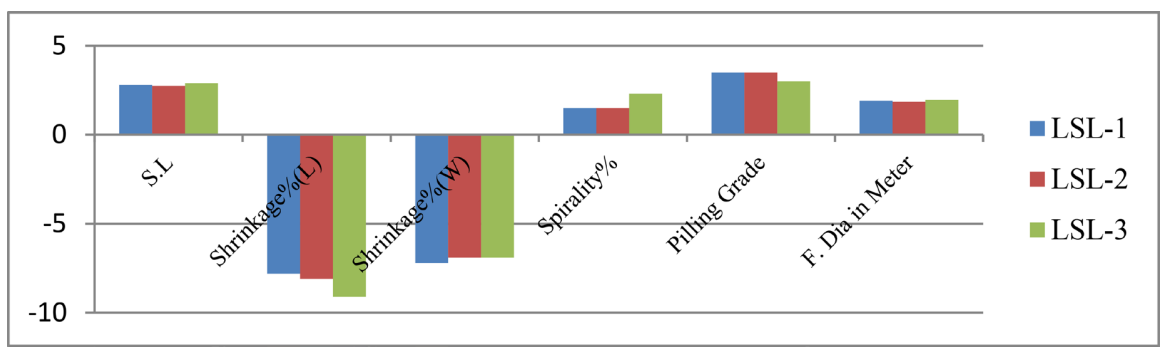

Figure 18. Yarn count \& stitch length effect on lycra single lacoste structure. 
length increases the pilling grade.

Comparison of knitted structure's GSM with or without Lycra (Figure 20).

Yarn count has a huge impact on the gram per square meter of fabric. Changing of yarn count, changes the GSM of specific structure. The finer the yarn count, the lower the fabric weight. The pie chart shows that lycra knitted structures produced more heavy fabric than knitted structures without lycra.

Normal and Lycra single jersey: Lycra is normally used in the knit cam of circular knitting machine during the production of any Lycra knitted structure. Lycra fabric is becoming more popular due to its suitability in the body. Lycra Single jersey requires more loose form than normal single jersey due to introduce the Lycra in the fabric. Finish width of normal single jersey is slightly less than the finish width of Lycra single jersey. Lengthwise and widthwise shrinkage\% of Lycra fabric is higher than normal fabric (Figure 21). The spirality\% of Lycra structure is also higher than normal structure (Figure 21). Pilling grade is dependent on the quality of yarn and compactness of the structure (Figure 21). The pilling grade of compact structure is higher than loose structure.

Normal and lycra single pique: The presence of Lycra in the knitted structure plays a very important role in deciding its technical parameter. The presence of Lycra in the knitted fabric impacts the S.L, Shrinkage, Finish width, Spirality and Pilling grade. The Lycra structure needed more loose form which decreases pilling grade. The maintenance of finish width of Lycra structure is comparatively more difficult than normal structure. The changes in the finish width change the technical parameter of the fabric. The deviation of finish width from

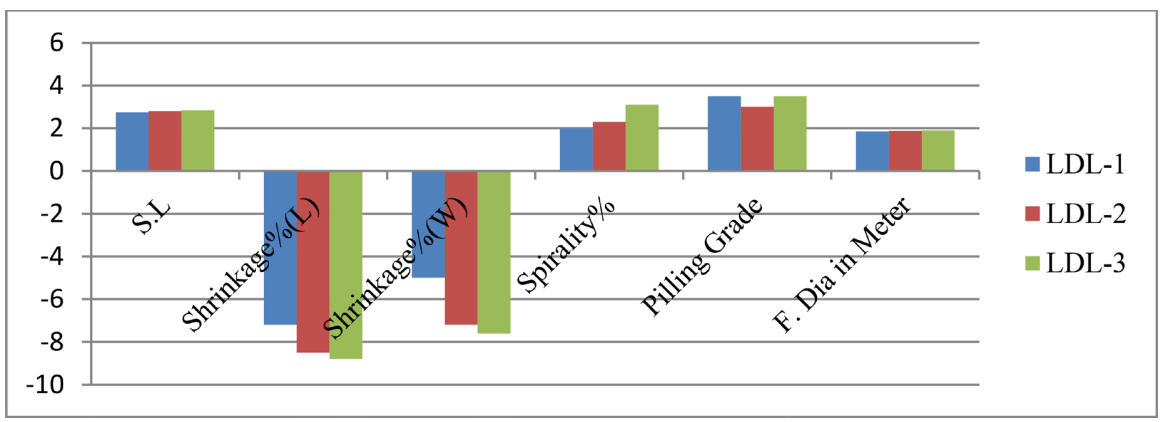

Figure 19. Yarn count \& stitch length effect on lycra double lacoste structure.

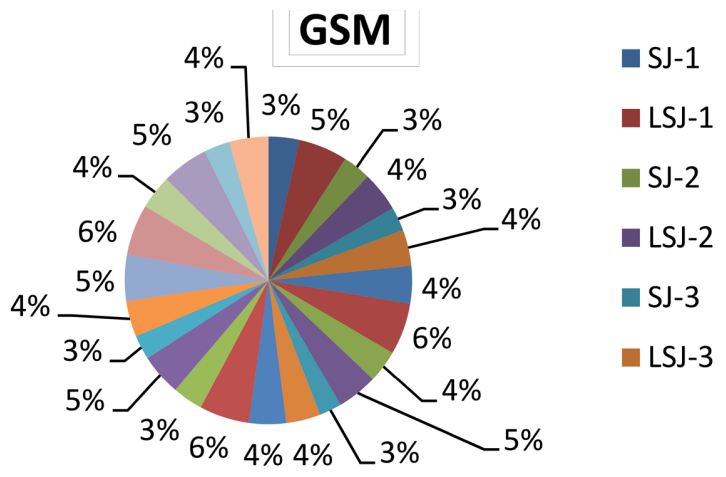

Figure 20. Yarn count effect on knitted structures. 


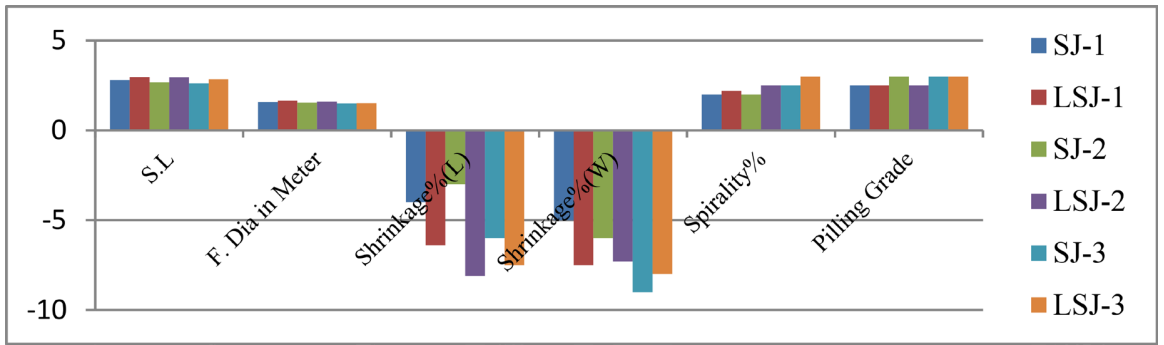

Figure 21. Comparison of normal and Lycra single jersey.

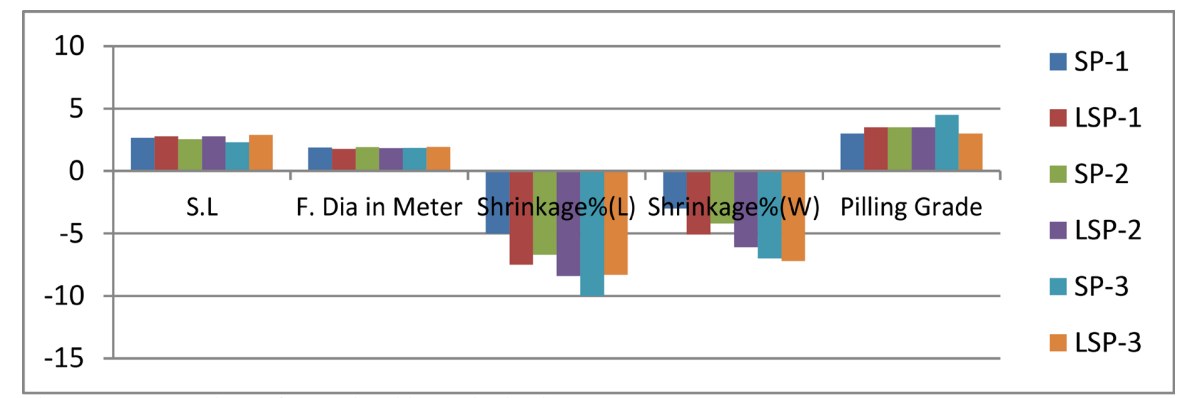

Figure 22. Comparison of normal and lycra single pique.

standard increases the shrinkage\% (Figure 22). So for controlling Shrinkage and spirality, it is also required to control finish diameter. The Lacoste structures show almost the same characteristics as normal and Lycra single pique.

Fabric structure has a significant impact on the GSM. When structure changes, GSM also changes for the same count.GSM increases around 10\% - 25\% for single pique, around $20 \%-35 \%$ for double pique, around $10 \%-25 \%$ for single lacoste and around $20 \%-40 \%$ for double lacoste in comparison with single jersey if dyeing and finishing parameter is maintained properly. It is also observed that the differences in GSM increase $\%$ also decreases with the increase of yarn count.

The presence of Lycra in the fabric structure increases the GSM about $40 \%$ $50 \%$ in comparison with Lycra single jersey and normal single jersey. The difference of GSM between Lycra single pique and normal piques is about 30\% $45 \%$ which is same for Lycra single lacoste and normal lacoste. GSM increases about 35\% - 50\% comparing Lycra double lacoste and normal double lacoste. In short, Lycra addition in the fabric structure increases the GSM about $30-50 \%$.

\section{Conclusion}

The parameters of knitted structure are largely controlled by yarn count, stitch length and finish dia. From the above data, it is proved that finish GSM mostly depends on yarn count and they are inversely related by each other. GSM gradually decreases and shrinkage\% increases when count increases. That's why finer count yarn is used to produce lower weight fabrics. Stitch length is another important parameter of knitted structure. More tightness or more looseness is not desirable in the structure. More loose or tighter form of fabric is more prone to increase shrinkage $\%$ and spirality $\%$ and loose form decrease pilling grade at the 
same time. Finish dia must need to control during the production of dyed fabric by stenter $\mathrm{m} / \mathrm{c}$. Finish dia deviation from standard is also responsible for low quality fabric. The lycra in the knitted structure adds another process named heat set before dyeing and finishing. The presence of lycra in the structure also increases GSM, S.L, shrinkage\% and spirality\% which was proved before. The productions of correct knitted structure maintaining all the parameters in acceptable level can only be possible when count yarn; stitch length and finish dia will strictly be maintained during the production.

\section{Acknowledgements}

We have been able to complete this program with the support and active cooperation of concerned bodies and authorities and several persons. We owe our dept and would like to express deep feelings of gratitude to our Knitting Manager Mr. Tanmoy Deb, Fabrics Manager Mr. Samrat and Knitting Master Mr. Ramiz Uddin, Amazing Fashion Limited, 10/1 Dagerchala, National University, Gazipur, Bangladesh. We are intensely indebted to our honorable Managing Director Engr. Md. A.L.M Ziaul Haque who selected us for accomplishing the program and for his overall support and motivation.

\section{Funding}

The author(s) disclosed receipt of the financial support for this paper, authorship: This program is funded by Amazing Fashion Limited, 10/1 dagerchala, National University, Gazipur, Dhaka, Bangladesh, Department of Research and Product Development.

\section{Conflicts of Interest}

The authors declare no conflicts of interest regarding the publication of this paper.

\section{References}

[1] Belal, S.A. (2009) Understanding Textiles for a Merchandiser. 2nd Edition, LB Graphics \& Printing, Bangladesh, 322, 383-394, 417-418.

[2] Burrow, S.W. and Nobile, D.P. (1993) Low Contamination Swab Employing Tubular Knit Fabrics. US5214821A.

[3] Spencer, D.J. (n.d.) Knitting Technology, A Comprehensive Handbook and Practical Guide. 3rd Edition, 105 p.

[4] Ramakrishna, S. (1997) Characterization and Modeling of the Tensile Properties of Plain Weft-Knit Fabrics-Reinforced Composites. Composites Science and Technology, 57, 1-22. https://doi.org/10.1016/S0266-3538(96)00098-X

[5] Rameshkumar, C., et al. (2008) Comparative Studies on Ring Rotor and Vortex Yarn Knitted Fabrics. Autex Research Journal, 8, 100-105.

[6] Rahman, H., Roy, P.K., Mian, L. and Saha, J. (2014) Effect of Lycra (Spandex) Percentage on Properties of Weft Knitted Single Jersey Fabric. Journal of Science and Technology, 4, 31-37. 
[7] https://www.google.com/search?q=properties+of+jersey\&oq=properties+of + jersey \&aqs=chrome.0i512j69i57j0i22i3018.17200j1j15\&sourceid $=$ chrome\&ie $=\mathrm{UTF}-8$

[8] Pique Knit Fabric. http://bosforustextile.com/pique_knit_fabric.html

[9] What Is Piqué Knit Fabric? https://silverbobbin.com/what-is-pique-knit-fabric/

[10] http://www.catwalkyourself.com/fashion-dictionary/elastan-espandex/

[11] Ray, S.C. (2012) Fundamentals and Advances in Knitting Technology. Woodhead Publishing India, Delhi, 199-212. https://doi.org/10.1533/9780857095558.199

[12] Senthilkumar, M., Anbumani, N. and de Araujo Universidade, M.D. (2011, August) Elastic Properties of Spandex Plated Cotton Knitted Fabrics. Journal of the Institution of Engineers (India), 92, 9-13.

[13] Voice, J., Dafniotis, P. and Towlson, S. (n.d.) Chapter 10: Elastic Textiles. In: Textiles in Sports, Wood Head Pulications, England, 205 p. 